\title{
Exact solutions of unsteady MHD free convection in a heat absorbing fluid flow past a flat plate with ramped wall temperature
}

\author{
Raj Nandkeolyar ${ }^{1}$, Mrutyunjay Das ${ }^{2}$ and Precious Sibanda ${ }^{1 *}$
}

\author{
"Correspondence: \\ sibandap@ukzn.ac.za \\ ${ }^{1}$ School of Mathematics, Statistics \\ and Computer Science, University \\ of KwaZulu-Natal, Scottsville, \\ Pietermaritzburg, 3209, South Africa \\ Full list of author information is \\ available at the end of the article
}

\begin{abstract}
Unsteady MHD free convection and mass transfer from a viscous, incompressible, electrically conducting and heat absorbing fluid flow past a vertical infinite flat plate is investigated. The flow is induced by a general time-dependent movement of the vertical plate, and the cases of ramped temperature and isothermal plates are studied. Exact solutions of the governing equations are obtained. The Sherwood number, Nusselt number and skin friction coefficients are obtained for both ramped temperature and isothermal plates. Some applications of practical interest are discussed for different types of plate motions. The numerical values of species concentration, fluid temperature and fluid velocity are displayed graphically whereas the numerical values of Sherwood number, the Nusselt number and skin friction are presented in tabular form, for different parameter values for both ramped and isothermal plates.
\end{abstract}

\section{Introduction}

The investigation of the effects of a magnetic field on the flow of a viscous, incompressible and electrically conducting fluid is important in many practical applications, such as in MHD power generators and boundary layer flow control. Due to this fact, a large number of researchers have contributed to the literature on the flow of fluids in the presence of a magnetic field. Hayat et al. [1] investigated the flow of a third-grade fluid on an oscillating porous plate in the presence of a transverse magnetic field. They obtained an analytic solution of the governing nonlinear boundary layer equations. Hayat et al. [2] also obtained the exact solution of an oscillatory boundary layer flow bounded by two horizontal flat plates, one of which was oscillating in its own plate and the other was at rest. Seth et al. [3] obtained the exact solution for the effects of Hall current on the rotating Hartmann flow in the presence of an inclined magnetic field.

Magnetohydrodynamic free convection finds applications in fluid engineering problems such as MHD pumps, accelerators and flow meters, plasma studies, nuclear reactors, geothermal energy extraction, etc. Free convective flow past a vertical plate in the presence of a transverse magnetic field has been studied by several researchers. Kim [4] studied the magnetohydrodynamic convective heat transfer past a semi-infinite vertical porous moving plate with variable suction. The combined effects of thermal and mass diffusion on the unsteady free convection flow of a viscous incompressible fluid over an

\section{黛 Springer}

○2013 Nandkeolyar et al.; licensee Springer. This is an Open Access article distributed under the terms of the Creative Commons Attribution License (http://creativecommons.org/licenses/by/2.0), which permits unrestricted use, distribution, and reproduction in any medium, provided the original work is properly cited. 
infinite vertical porous plate was investigated by Takhar et al. [5]. Ahmed et al. [6] considered the effects of thermal diffusion on a three-dimensional MHD free convection flow of a viscous incompressible fluid over a vertical plate embedded in a porous medium.

The combined effects of convective heat and mass transfer on the flow of a viscous, incompressible and electrically conducting fluid has many engineering and geophysical applications such as in geothermal reservoirs, drying of porous solids, thermal insulation, enhanced oil recovery, cooling of nuclear reactor and underground energy transports. The hydromagnetic free convection flow with mass transfer effect has been studied extensively by many researchers. Hossain and Mandal [7] discussed the mass transfer effects on the unsteady hydromagnetic free convection flow past an accelerated vertical porous plate. Jha [8] investigated the hydromagnetic free convection flow through a porous medium with mass transfer. Elbashbeshy [9] studied the heat and mass transfer along a vertical plate with variable surface tension and concentration in the presence of a magnetic field. Chamkha and Khaled [10] investigated the hydromagnetic combined heat and mass transfer by natural convection from a permeable surface embedded in a fluid saturated porous medium. Chen [11] studied the combined heat and mass transfer in MHD free convection from a vertical surface with Ohmic heating and viscous dissipation. Afify [12] discussed the MHD free convective heat and mass transfer flow over a stretching sheet in the presence of suction/injection with thermal diffusion and diffusion thermo effects. Eldabe et al. [13] studied the unsteady motion of an MHD viscous incompressible fluid with heat and mass transfer through porous medium near a moving vertical plate.

Heat absorption/generation effects have significant impact on the heat and mass transfer flow of a viscous, incompressible and electrically conducting fluid. Chamkha and Khaled [14] investigated heat generation/absorption effects on hydromagnetic combined heat and mass transfer flow from an inclined plate. The effects of a heat source/sink on unsteady MHD convection through porous medium with combined heat and mass transfer was studied by Kamel [15]. Chamkha [16] solved the problem of unsteady MHD convective heat and mass transfer past a semi-infinite vertical permeable moving plate with heat absorption. Makinde [17] discussed the hydromagnetic boundary layer flow and mass transfer past a vertical plate in a porous medium with constant heat flux.

In most of the above investigations, the solutions were obtained by assuming the velocity and temperature at the interface to be continuous and well defined. There are, however, several problems of physical interest that may require non-uniform or arbitrary wall conditions. Several researchers (e.g., [18-21]) have investigated problems of free convection from a vertical plate with step-discontinuities in the surface temperature. Chandran et al. [22] studied the unsteady natural convection flow in a viscous incompressible fluid near a vertical plate with ramped wall temperature. The MHD natural convection flow past an impulsively moving vertical plate with ramped wall temperature in the presence of thermal diffusion with heat absorption was studied by Seth and Ansari [23]. Recently, Seth et al. [24] studied the unsteady natural convection flow of a viscous incompressible electrically conducting fluid past an impulsively moving vertical plate in a porous medium with ramped wall temperature taking into account the effects of thermal radiation. They compared the results of natural convection near a ramped temperature plate with those of natural convection near an isothermal plate.

The aim of the present paper is to study the hydromagnetic free convective heat and mass transfer flow of a viscous, incompressible, electrically conducting and heat absorbing 
fluid past a vertical infinite flat plate. The fluid flow is induced by a general time-dependent movement of the infinite plate. The governing equations are solved analytically, and a general solution valid for any time-dependent movement of the plate is obtained. Some particular cases that highlight the applications of the general solution are discussed.

\section{Formulation of the problem}

Consider the unsteady MHD free convection heat and mass transfer flow of a viscous, incompressible, electrically conducting and heat absorbing fluid along an infinite nonconducting vertical flat plate. The $x$-axis is along the plate in the upward direction, the $y$-axis normal to it and the $z$-axis normal to $x y$-plane. The fluid is permeated by a uniform transverse magnetic field of strength $B_{0}$ applied along the $y$-axis. For time $t^{\prime}<0$, the stationary plate and the fluid are at the same constant temperature $T_{\infty}^{\prime}$ and species concentration $C_{\infty}^{\prime}$. At time $t^{\prime}=0$, the plate begins to move with a time-dependent velocity $U_{0} f\left(t^{\prime}\right)$ in its own plane along the $x$-axis and the temperature of the plate is raised or lowered to $T_{\infty}^{\prime}+\left(T_{w}^{\prime}-T_{\infty}^{\prime}\right) t^{\prime} / t_{0}$ when $t^{\prime}<t_{0}$ and thereafter, for $t^{\prime}>t_{0}$, it is maintained at a uniform temperature $T_{w}^{\prime}$. Also, for time $t^{\prime}>0$, species concentration is raised to $C_{w}^{\prime}$. The geometry of the problem is presented in Figure 1. Since the plate is of infinite extent in $x$ and $z$ directions, and is electrically non-conducting, all physical quantities, except pressure, are functions of $y$ and $t^{\prime}$ only.

The fluid under consideration is a metallic liquid, such as mercury, whose magnetic Reynolds number is small, and hence the induced magnetic field produced by the fluid motion is negligible in comparison to the applied magnetic field $\mathbf{B} \equiv\left(0, B_{0}, 0\right)$, Cramer and Pai [25]. Also, no external electric field is applied, so the effect of polarization of the magnetic field is negligible, Meyer [26]. With these assumptions, the governing equations for the unsteady MHD free convection flow and mass transfer of a viscous, incompressible, electrically conducting and heat absorbing fluid are as follows:

$$
\begin{aligned}
\frac{\partial u^{\prime}}{\partial t^{\prime}} & =v \frac{\partial^{2} u^{\prime}}{\partial y^{2}}-\frac{\sigma B_{0}^{2}}{\rho} u^{\prime}+g \beta^{*}\left(T^{\prime}-T_{\infty}^{\prime}\right)+g \beta^{\prime}\left(C^{\prime}-C_{\infty}^{\prime}\right), \\
\frac{\partial T^{\prime}}{\partial t^{\prime}} & =\frac{k}{\rho c_{p}} \frac{\partial^{2} T^{\prime}}{\partial y^{2}}-\frac{Q_{0}}{\rho c_{p}}\left(T^{\prime}-T_{\infty}^{\prime}\right), \\
\frac{\partial C^{\prime}}{\partial t^{\prime}} & =D \frac{\partial^{2} C^{\prime}}{\partial y^{2}},
\end{aligned}
$$

where $u^{\prime}, \rho, g, \beta^{*}, \beta^{\prime}, T^{\prime}, C^{\prime}, c_{p}, k, v, \sigma, D$ and $Q_{0}$ are, respectively, the fluid velocity in the $x$-direction, the fluid density, acceleration due to gravity, the volumetric coefficient of thermal expansion, the volumetric coefficient of expansion for concentration, the temperature of the fluid, species concentration, specific heat at constant pressure, thermal conductivity,

Figure 1 Geometry of the problem.

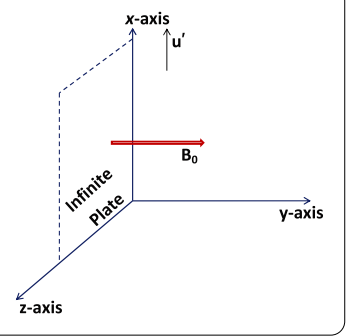


the kinematic coefficient of viscosity, electrical conductivity, chemical molecular diffusivity and the heat absorption coefficient. Assuming no slip between the plate and the fluid, the initial and boundary conditions are

$$
\begin{aligned}
& u^{\prime}=0, \quad T^{\prime}=T_{\infty}^{\prime}, \quad C^{\prime}=C_{\infty}^{\prime} \quad \text { for } y \geq 0 \text { and } t^{\prime} \leq 0, \\
& u^{\prime}=U_{0} f\left(t^{\prime}\right), \quad C^{\prime}=C_{w}^{\prime} \quad \text { at } y=0 \text { for } t^{\prime}>0, \\
& T^{\prime}=T_{\infty}^{\prime}+\left(T_{w}^{\prime}-T_{\infty}^{\prime}\right) t^{\prime} / t_{0} \quad \text { at } y=0 \text { for } 0<t^{\prime} \leq t_{0}, \\
& T^{\prime}=T_{w}^{\prime} \quad \text { at } y=0 \text { for } t^{\prime}>t_{0}, \\
& u^{\prime} \rightarrow 0, \quad T^{\prime} \rightarrow T_{\infty}^{\prime}, \quad C^{\prime} \rightarrow C_{\infty}^{\prime} \quad \text { as } y \rightarrow \infty \text { for } t^{\prime}>0 .
\end{aligned}
$$

Introducing the following non-dimensional variables

$$
\eta=\frac{y}{U_{0} t_{0}}, \quad u=\frac{u^{\prime}}{U_{0}}, \quad t=\frac{t^{\prime}}{t_{0}}, \quad T=\frac{T^{\prime}-T_{\infty}^{\prime}}{T_{w}^{\prime}-T_{\infty}^{\prime}} \quad \text { and } \quad C=\frac{C^{\prime}-C_{\infty}^{\prime}}{C_{w}^{\prime}-C_{\infty}^{\prime}},
$$

equations (1)-(3) reduce to

$$
\begin{aligned}
& \frac{\partial u}{\partial t}=\frac{\partial^{2} u}{\partial \eta^{2}}+G r T+G m C-M u \\
& \frac{\partial T}{\partial t}=\frac{1}{\operatorname{Pr}} \frac{\partial^{2} T}{\partial \eta^{2}}-\phi T \\
& \frac{\partial C}{\partial t}=\frac{1}{S c} \frac{\partial^{2} C}{\partial \eta^{2}}
\end{aligned}
$$

where $M=\sigma B_{0}^{2} \nu / \rho U_{0}^{2}$ is the magnetic parameter, $G r=g \beta^{*} \nu\left(T_{w}^{\prime}-T_{\infty}^{\prime}\right) / U_{0}^{3}$ is the thermal Grashof number, $G m=g \beta^{\prime} v\left(C_{w}^{\prime}-C_{\infty}^{\prime}\right) / U_{0}^{3}$ is the mass Grashof number, $P r=\rho v c_{p} / k$ is the Prandtl number, $S c=v / D$ is the Schmidt number and $\phi=\nu Q_{0} / \rho c_{p} U_{0}^{2}$ is the heat absorption parameter. The characteristic time $t_{0}$ is defined as

$$
t_{0}=\frac{v}{U_{0}^{2}}
$$

The corresponding initial and boundary conditions in non-dimensional form become

$$
\begin{aligned}
& u=0, \quad T=0, \quad C=0 \quad \text { for } \eta \geq 0 \text { and } t \leq 0, \\
& u=f(t), \quad C=1 \quad \text { at } \eta=0 \text { for } t>0, \\
& T=t \quad \text { at } \eta=0 \text { for } 0<t \leq 1, \\
& T=1 \quad \text { at } \eta=0 \text { for } t>1, \\
& u \rightarrow 0, \quad T \rightarrow 0, \quad C \rightarrow 0 \quad \text { as } \eta \rightarrow \infty \text { for } t>0 .
\end{aligned}
$$

The system of differential equations (5)-(7) together with the initial and boundary conditions (9a)-(9e) describes our model for the MHD free convective heat and mass transfer flow of a viscous, incompressible, electrically conducting and heat absorbing fluid past a vertical flat plate with ramped wall temperature. 


\section{Solution of the problem}

The set of equations (5)-(7) subject to the initial and boundary conditions (9a)-(9e) were solved analytically using Laplace transforms. The exact solutions for species concentration $C(\eta, t)$, fluid temperature $T(\eta, t)$ and fluid velocity $u(\eta, t)$ are, respectively,

$$
\begin{aligned}
C(\eta, t) & =\operatorname{erfc}\left(\frac{\eta}{2} \sqrt{\frac{a}{t}}\right), \\
T(\eta, t) & =P_{1}(\eta, t)-H(t-1) P_{1}(\eta, t-1), \\
u(\eta, t) & =P(\eta, t)+\alpha_{1}\left[P_{2}(\eta, t)-H(t-1) P_{2}(\eta, t-1)\right]+\alpha_{2} P_{3}(\eta, t),
\end{aligned}
$$

where

$$
\begin{aligned}
& P(\eta, t)=\mathcal{L}^{-1}\left[\bar{f}(s) e^{-\eta \sqrt{s+M}}\right], \\
& P_{1}(\eta, t)=\left(\frac{t}{2}+\frac{c \eta}{4 \sqrt{d}}\right) e^{\eta \sqrt{d}} \operatorname{erfc}\left(\mathrm{t}_{1}\right)+\left(\frac{t}{2}-\frac{c \eta}{4 \sqrt{d}}\right) e^{-\eta \sqrt{d}} \operatorname{erfc}\left(\mathrm{t}_{2}\right), \\
& P_{2}(\eta, t)=\left(\frac{\alpha t-1}{2 \alpha^{2}}+\frac{c \eta}{4 \alpha \sqrt{d}}\right) e^{\eta \sqrt{d}} \operatorname{erfc}\left(\mathrm{t}_{1}\right)+\left(\frac{\alpha t-1}{2 \alpha^{2}}-\frac{c \eta}{4 \alpha \sqrt{d}}\right) e^{-\eta \sqrt{d}} \operatorname{erfc}\left(\mathrm{t}_{2}\right) \\
& +\frac{e^{-\alpha t}}{2 \alpha^{2}}\left[e^{\eta \sqrt{d-\alpha c}} \operatorname{erfc}\left(\mathrm{t}_{3}\right)+e^{-\eta \sqrt{d-\alpha c}} \operatorname{erfc}\left(\mathrm{t}_{4}\right)\right] \\
& -\left(\frac{\alpha t-1}{2 \alpha^{2}}+\frac{\eta}{4 \alpha \sqrt{M}}\right) e^{\eta \sqrt{M}} \operatorname{erfc}\left(\mathrm{t}_{5}\right)-\left(\frac{\alpha t-1}{2 \alpha^{2}}-\frac{\eta}{4 \alpha \sqrt{M}}\right) e^{-\eta \sqrt{M}} \operatorname{erfc}\left(\mathrm{t}_{6}\right) \\
& -\frac{e^{-\alpha t}}{2 \alpha^{2}}\left[e^{\eta \sqrt{M-\alpha}} \operatorname{erfc}\left(\mathrm{t}_{7}\right)+e^{-\eta \sqrt{M-\alpha}} \operatorname{erfc}\left(\mathrm{t}_{8}\right)\right] \text {, } \\
& P_{3}(\eta, t)=\frac{1}{\beta} \operatorname{erfc}\left(\mathrm{t}_{9}\right)-\frac{e^{-\beta t}}{2 \beta}\left[e^{\eta \sqrt{-a \beta}} \operatorname{erfc}\left(\mathrm{t}_{10}\right)+e^{-\eta \sqrt{-a \beta}} \operatorname{erfc}\left(\mathrm{t}_{11}\right)\right] \\
& -\frac{1}{2 \beta}\left[e^{\eta \sqrt{M}} \operatorname{erfc}\left(\mathrm{t}_{5}\right)+e^{-\eta \sqrt{M}} \operatorname{erfc}\left(\mathrm{t}_{6}\right)\right] \\
& +\frac{e^{-\beta t}}{2 \beta}\left[e^{\eta \sqrt{M-\beta}} \operatorname{erfc}\left(\mathrm{t}_{12}\right)+e^{-\eta \sqrt{M-\beta}} \operatorname{erfc}\left(\mathrm{t}_{13}\right)\right], \\
& \mathrm{t}_{1}, \mathrm{t}_{2}= \pm \sqrt{\frac{d t}{c}}+\frac{\eta}{2} \sqrt{\frac{c}{t}}, \quad \mathrm{t}_{3}, \mathrm{t}_{4}= \pm \sqrt{\frac{(d-\alpha c) t}{c}}+\frac{\eta}{2} \sqrt{\frac{c}{t}}, \\
& \mathrm{t}_{5}, \mathrm{t}_{6}= \pm \sqrt{M t}+\frac{\eta}{2 \sqrt{t}}, \quad \mathrm{t}_{7}, \mathrm{t}_{8}= \pm \sqrt{(M-\alpha) t}+\frac{\eta}{2 \sqrt{t}}, \\
& \mathrm{t}_{9}=\frac{\eta}{2} \sqrt{\frac{a}{t}}, \quad \mathrm{t}_{10}, \mathrm{t}_{11}= \pm \sqrt{-\beta t}+\frac{\eta}{2} \sqrt{\frac{a}{t}}, \quad \mathrm{t}_{12}, \mathrm{t}_{13}= \pm \sqrt{(M-\beta) t}+\frac{\eta}{2 \sqrt{t}}, \\
& a=S c, \quad c=\operatorname{Pr}, \quad d=\operatorname{Pr} \phi, \quad \alpha_{1}=\frac{-G r}{c-1}, \quad \alpha_{2}=\frac{-G m}{a-1}, \\
& \alpha=\frac{d-M}{c-1} \text { and } \beta=\frac{-M}{a-1} \text {. }
\end{aligned}
$$

Here, $\operatorname{erfc}(x), \mathcal{L}^{-1}$ and $H(t-1)$ are respectively the complimentary error function, the inverse Laplace transform operator and the Heaviside unit step function.

Equations (10)-(12) represent the analytical solutions for the free convective heat and mass transfer flow of a viscous, incompressible, electrically conducting and heat absorbing 
fluid past a flat plate with ramped temperature in the presence of a uniform transverse magnetic field. In order to highlight the effects of the ramped temperature on the fluid flow, it is worthwhile to compare such a flow with the flow near a moving plate with constant temperature. The solution for species concentration is given by equation (10). However, the fluid temperature and velocity for free convection near an isothermal plate has the following form:

$$
\begin{aligned}
& T(\eta, t)=\frac{1}{2}\left[e^{\eta \sqrt{d}} \operatorname{erfc}\left(\mathrm{t}_{1}\right)+e^{-\eta \sqrt{d}} \operatorname{erfc}\left(\mathrm{t}_{2}\right)\right], \\
& u(\eta, t)=P(\eta, t)+\alpha_{2} P_{3}(\eta, t)+\alpha_{1} P_{4}(\eta, t),
\end{aligned}
$$

where

$$
\begin{aligned}
P_{4}(\eta, t)= & \frac{1}{2 \alpha}\left[e^{\eta \sqrt{d}} \operatorname{erfc}\left(\mathrm{t}_{1}\right)+e^{-\eta \sqrt{d}} \operatorname{erfc}\left(\mathrm{t}_{2}\right)\right] \\
& -\frac{e^{-\alpha t}}{2 \alpha}\left[e^{\eta \sqrt{(d-\alpha c)}} \operatorname{erfc}\left(\mathrm{t}_{3}\right)+e^{-\eta \sqrt{(d-\alpha c)}} \operatorname{erfc}\left(\mathrm{t}_{4}\right)\right] \\
& -\frac{1}{2 \alpha}\left[e^{\eta \sqrt{M}} \operatorname{erfc}\left(\mathrm{t}_{5}\right)+e^{-\eta \sqrt{M}} \operatorname{erfc}\left(\mathrm{t}_{6}\right)\right] \\
& +\frac{e^{-\alpha t}}{2 \alpha}\left[e^{\eta \sqrt{M-\alpha}} \operatorname{erfc}\left(\mathrm{t}_{7}\right)+e^{-\eta \sqrt{M-\alpha}} \operatorname{erfc}\left(\mathrm{t}_{8}\right)\right] .
\end{aligned}
$$

The physical quantities of engineering interest are the Sherwood number Sh, the Nusselt number $N u$ and skin-friction $\tau$. The Sherwood number measures the rate of mass transfer at the plate and is given by

$$
S h=-\left(\frac{\partial C}{\partial \eta}\right)_{\eta=0}=\sqrt{\frac{a}{\pi t}} .
$$

The Nusselt number measures the rate of heat transfer at the plate, and for a ramped temperature plate it is

$$
N u=-\left(\frac{\partial T}{\partial \eta}\right)_{\eta=0}=-\left[F_{1}(t)-H(t-1) F_{1}(t-1)\right],
$$

where

$$
F_{1}(t)=-\left(\sqrt{d} t+\frac{c}{2 \sqrt{d}}\right) \operatorname{erf}\left(\sqrt{\frac{d t}{c}}\right)-\sqrt{\frac{c t}{\pi}} e^{-\frac{d t}{c}} .
$$

In the case of an isothermal plate, the Nusselt number is

$$
N u_{i}=(\sqrt{d}) \operatorname{erf}\left(\sqrt{\frac{d t}{c}}\right)+\sqrt{\frac{c}{\pi t}} e^{-\frac{d t}{c}}
$$

Equations (10)-(14) represent the analytical solutions for the flow induced by a general time-dependent movement of the vertical flat plate. To gain some practical understanding of the flow dynamics, some particular cases of time-dependent movements of the plate are discussed below. 


\subsection{Plate movement with uniform velocity}

Assuming that the plate moves with uniform velocity $f(t)=H(t)$, the fluid velocity for the ramped temperature plate is obtained as

$$
u(\eta, t)=P_{5}(\eta, t)+\alpha_{1}\left[P_{2}(\eta, t)-H(t-1) P_{2}(\eta, t-1)\right]+\alpha_{2} P_{3}(\eta, t),
$$

while the isothermal plate has the velocity

$$
u(\eta, t)=P_{5}(\eta, t)+\alpha_{1} P_{4}(\eta, t)+\alpha_{2} P_{3}(\eta, t),
$$

where

$$
P_{5}(\eta, t)=\frac{1}{2}\left[e^{\eta \sqrt{M}} \operatorname{erfc}\left(\mathrm{t}_{5}\right)+e^{-\eta \sqrt{M}} \operatorname{erfc}\left(\mathrm{t}_{6}\right)\right] .
$$

The skin friction for the ramped temperature plate is

$$
\tau_{1}=\left(\frac{\partial u}{\partial \eta}\right)_{\eta=0}=Q_{1}(t)+\alpha_{1}\left[F_{2}(t)-H(t-1) F_{2}(t-1)\right]+\alpha_{2} F_{3}(t),
$$

and for the isothermal plate

$$
\tau_{1 i}=Q_{1}(t)+\alpha_{1} F_{4}(t)+\alpha_{2} F_{3}(t),
$$

where

$$
\begin{aligned}
Q_{1}(t)= & -\sqrt{M} \operatorname{erf}(\sqrt{M t})-\frac{e^{-M t}}{\sqrt{\pi t}}, \\
F_{2}(t)= & -\left[\left(\frac{\alpha t-1}{\alpha^{2}}\right) \sqrt{d}+\frac{c}{2 \alpha \sqrt{d}}\right] \operatorname{erf}\left(\sqrt{\frac{d t}{c}}\right)-\frac{\sqrt{d-\alpha c}}{\alpha^{2}} \operatorname{erf}\left(\sqrt{\left(\frac{d-\alpha c}{c}\right) t}\right) e^{-\alpha t} \\
& +\left[\left(\frac{\alpha t-1}{\alpha^{2}}\right) \sqrt{M}+\frac{1}{2 \alpha \sqrt{M}}\right] \operatorname{erf}(\sqrt{M t})+\frac{\sqrt{M-\alpha}}{\alpha^{2}} \operatorname{erf}(\sqrt{(M-\alpha) t}) e^{-\alpha t} \\
& -\frac{e^{-\frac{d t}{c}}}{\alpha} \sqrt{\frac{c t}{\pi}}+\frac{e^{-M t}}{\alpha} \sqrt{\frac{t}{\pi}}, \\
F_{3}(t)= & \frac{1}{\beta}\left[\sqrt{-a \beta} \operatorname{erf}(\sqrt{-\beta t}) e^{-\beta t}+\sqrt{M} \operatorname{erf}(\sqrt{M t})-\sqrt{M-\beta} \operatorname{erf}(\sqrt{(M-\beta) t}) e^{-\beta t}\right], \\
F_{4}(t)= & \frac{-\sqrt{d}}{\alpha} \operatorname{erf}\left(\sqrt{\frac{d t}{c}}\right)+\frac{\sqrt{d-\alpha c}}{\alpha} \operatorname{erf}\left(\sqrt{\left(\frac{d-\alpha c}{c}\right) t}\right) e^{-\alpha t}+\frac{\sqrt{M}}{\alpha} \operatorname{erf}(\sqrt{M t}) \\
& -\frac{\sqrt{M-\alpha}}{\alpha} \operatorname{erf}(\sqrt{(M-\alpha) t}) e^{-\alpha t} .
\end{aligned}
$$

The solutions (11), (13), (17), (19), (20), (21), (23) and (24), in the absence of heat absorption $(\phi=0)$ and mass transfer $(G m=0)$, are in agreement with the results obtained by Seth $e t$ al. [24] when $K_{1}=0, N=0$. The results are also consistent with those of Seth and Ansari [23] for a non-porous medium $K_{1}=0$ in the absence of mass transfer and with the results reported by Chandran et al. [22] in the absence of a magnetic field $M=0$, mass transfer and heat absorption. 


\subsection{Plate movement with single acceleration}

Assuming that the plate moves with single acceleration $f(t)=t H(t)$, the fluid velocity for a ramped temperature plate was obtained as

$$
u(\eta, t)=P_{6}(\eta, t)+\alpha_{1}\left[P_{2}(\eta, t)-H(t-1) P_{2}(\eta, t-1)\right]+\alpha_{2} P_{3}(\eta, t),
$$

and for the isothermal plate

$$
u(\eta, t)=P_{6}(\eta, t)+\alpha_{1} P_{4}(\eta, t)+\alpha_{2} P_{3}(\eta, t),
$$

where

$$
P_{6}(\eta, t)=\left(\frac{t}{2}+\frac{\eta}{4 \sqrt{M}}\right) e^{\eta \sqrt{M}} \operatorname{erfc}\left(\mathrm{t}_{5}\right)+\left(\frac{t}{2}-\frac{\eta}{4 \sqrt{M}}\right) e^{-\eta \sqrt{M}} \operatorname{erfc}\left(\mathrm{t}_{6}\right) .
$$

The skin friction for the ramped temperature plate is

$$
\tau_{2}=Q_{2}(t)+\alpha_{1}\left[F_{2}(t)-H(t-1) F_{2}(t-1)\right]+\alpha_{2} F_{3}(t) .
$$

The corresponding skin friction for the isothermal plate is

$$
\tau_{2 i}=Q_{2}(t)+\alpha_{1} F_{4}(t)+\alpha_{2} F_{3}(t)
$$

where

$$
Q_{2}(t)=-\left(\frac{1}{2 \sqrt{M}}+t \sqrt{M}\right) \operatorname{erf}(\sqrt{M t})-\sqrt{\frac{t}{\pi}} e^{-M t} .
$$

\subsection{Plate movement with periodic acceleration}

Assuming that the plate moves with periodic acceleration $f(t)=\cos \omega t H(t)$, the fluid velocity for the ramped temperature plate is

$$
u(\eta, t)=P_{7}(\eta, t)+\alpha_{1}\left[P_{2}(\eta, t)-H(t-1) P_{2}(\eta, t-1)\right]+\alpha_{2} P_{3}(\eta, t)
$$

and the fluid velocity for the isothermal plate is

$$
u(\eta, t)=P_{7}(\eta, t)+\alpha_{1} P_{4}(\eta, t)+\alpha_{2} P_{3}(\eta, t),
$$

where

$$
\begin{aligned}
P_{7}(\eta, t)= & \frac{1}{4} e^{-i \omega t}\left[e^{\eta \sqrt{M-i \omega}} \operatorname{erfc}\left(\sqrt{(M-i \omega) t}+\frac{\eta}{2 \sqrt{t}}\right)\right. \\
& \left.+e^{-\eta \sqrt{M-i \omega}} \operatorname{erfc}\left(-\sqrt{(M-i \omega) t}+\frac{\eta}{2 \sqrt{t}}\right)\right] \\
& +\frac{1}{4} e^{i \omega t}\left[e^{\eta \sqrt{M+i \omega}} \operatorname{erfc}\left(\sqrt{(M+i \omega) t}+\frac{\eta}{2 \sqrt{t}}\right)\right. \\
& \left.+e^{-\eta \sqrt{M+i \omega}} \operatorname{erfc}\left(-\sqrt{(M+i \omega) t}+\frac{\eta}{2 \sqrt{t}}\right)\right] .
\end{aligned}
$$


The skin friction for the ramped temperature plate is expressed as

$$
\tau_{3}=Q_{3}(t)+\alpha_{1}\left[F_{2}(t)-H(t-1) F_{2}(t-1)\right]+\alpha_{2} F_{3}(t),
$$

and the skin friction for the isothermal plate is

$$
\tau_{3 i}=Q_{3}(t)+\alpha_{1} F_{4}(t)+\alpha_{2} F_{3}(t)
$$

where

$$
\begin{aligned}
Q_{3}(t)= & -\frac{e^{-M t}}{\sqrt{\pi t}}-\frac{1}{2} \sqrt{M-i \omega} e^{-i \omega t} \operatorname{erf}(\sqrt{(M-i \omega) t}) \\
& -\frac{1}{2} \sqrt{M+i \omega} e^{i \omega t} \operatorname{erf}(\sqrt{(M+i \omega) t}) .
\end{aligned}
$$

\section{Results and discussion}

The effects of various flow parameters on the MHD heat and mass transfer in the fluid flow past a flat plate, the velocity, species concentration and temperature profiles are given in Figures 2-10 when $\omega=\pi / 2$ for both ramped temperature and isothermal plates. The values of the skin friction, Nusselt number and Sherwood number are presented in Tables 1-4, again for both ramped temperature and isothermal plates.

Figure 2 shows that species concentration $C(\eta, t)$ increases with $t$ and decreases with an increase in $S c$. Since the $S c h m i d t$ number $S c$ is the ratio of viscosity to mass diffusivity, an increase in $S c$ implies a decrease in the mass diffusion rate. Thus it follows that species concentration increases with an increase in time or mass diffusion rate.

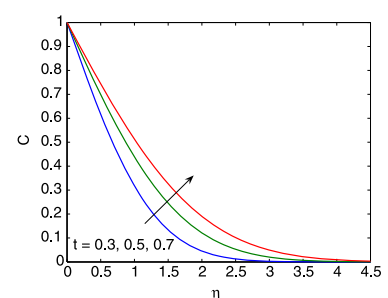

(a)

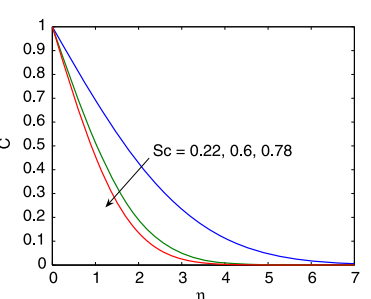

(b)

Figure 2 Effect of (a) time $t$ and (b) the Schmidt number Sc on species concentration $C(\eta, t)$.

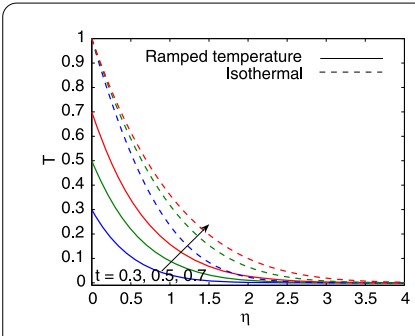

(a)

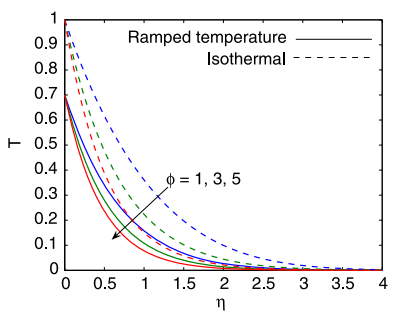

(b)

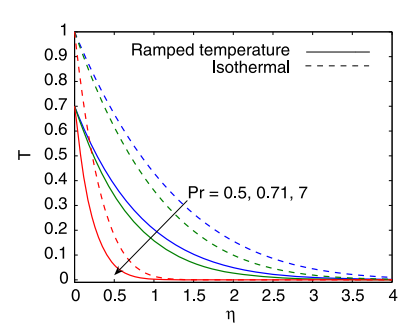

(c)

Figure 3 Effect of variation in (a) time $t$, (b) the heat absorption parameter $\phi$, and (c) the Prandtl number $\operatorname{Pr}$ on temperature $T(\eta, t)$. 


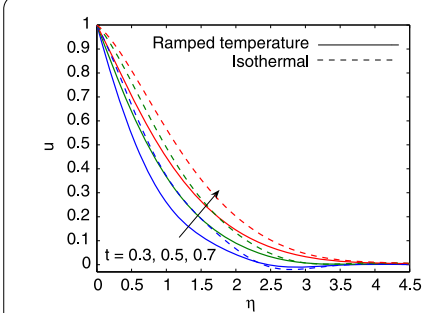

(a)

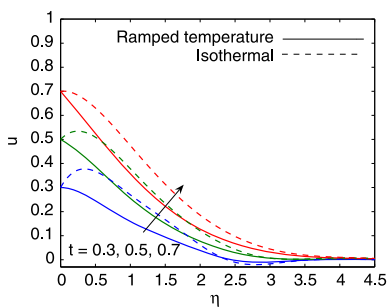

(b)

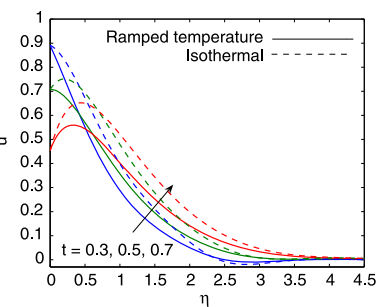

(c)

Figure 4 Effect of variation in time $t$ on the fluid velocity $u(\eta, t)$ when (a) $f(t)=H(t),(b) f(t)=t H(t)$, and (c) $f(t)=\cos \omega t H(t)$ for $S c=0.6, \phi=1, \operatorname{Pr}=0.71, M=3, G r=2$ and $G m=2$.

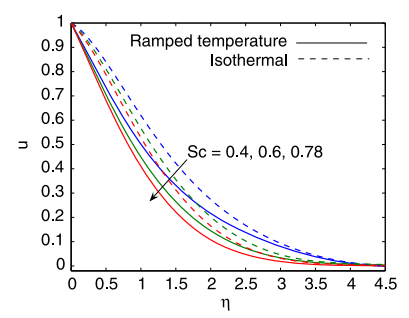

(a)

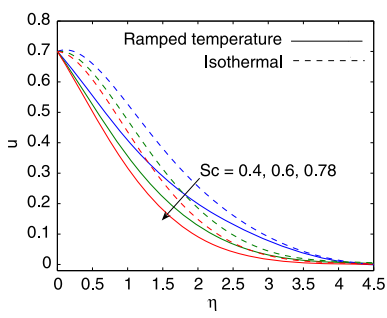

(b)

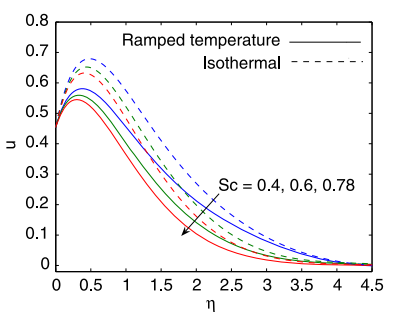

(c)

Figure 5 Effect of variation in the Schmidt number Sc on the fluid velocity $u(\eta, t)$ when (a) $f(t)=H(t)$, (b) $f(t)=t H(t)$, and (c) $f(t)=\cos \omega t H(t)$ for $t=0.7, \phi=1, P r=0.71, M=3, G r=2$ and $G m=2$.

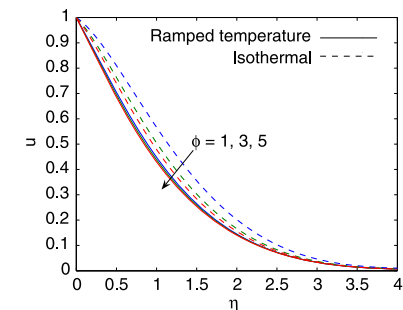

(a)

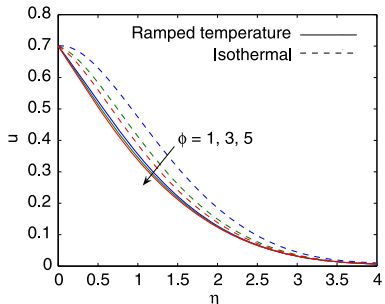

(b)

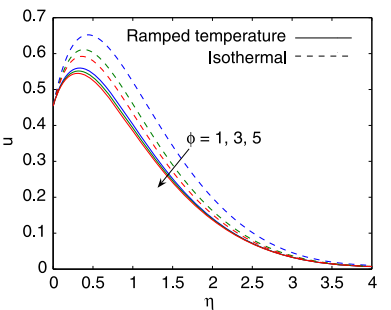

(c)

Figure 6 Effect of variation in the heat absorption parameter $\phi$ on the fluid velocity $u(\eta, t)$ when (a) $f(t)=H(t)$, (b) $f(t)=t H(t)$, and (c) $f(t)=\cos \omega t H(t)$ for $t=0.7, S c=0.6, P r=0.71, M=3, G r=2$ and $G m=2$.

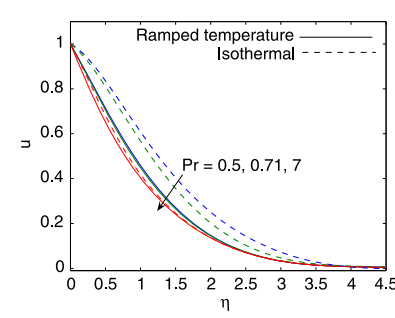

(a)

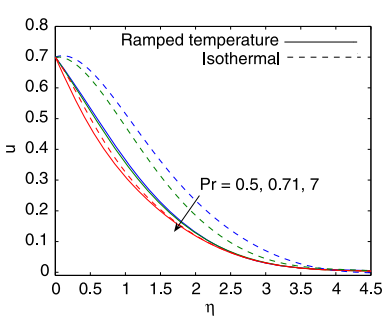

(b)

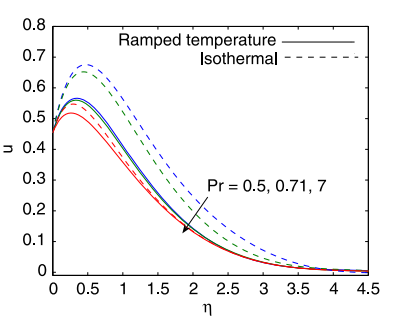

(c)

Figure 7 Effect of variation in the Prandtl number $\operatorname{Pr}$ on the fluid velocity $u(\eta, t)$ when (a) $f(t)=H(t)$, (b) $f(t)=t H(t)$, and (c) $f(t)=\cos \omega t H(t)$ for $t=0.7, S c=0.6, \phi=1, M=3, G r=2$ and $G m=2$. 


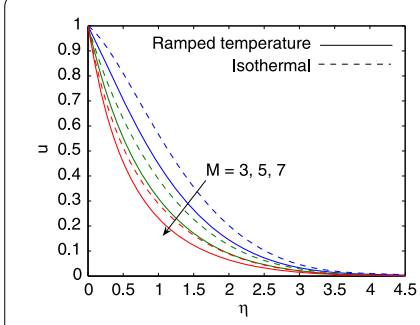

(a)

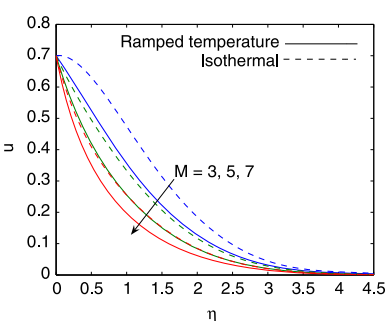

(b)

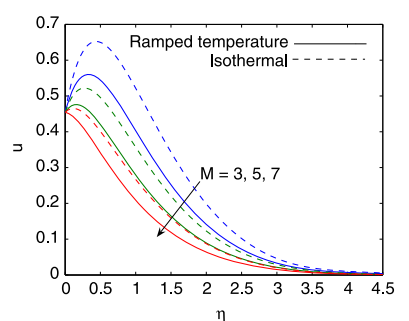

(c)

Figure 8 Effect of variation in the magnetic parameter $M$ on the fluid velocity $u(\eta, t)$ when (a) $f(t)=H(t)$, (b) $f(t)=t H(t)$, and (c) $f(t)=\cos \omega t H(t)$ for $t=0.7, S c=0.6, \phi=1, \operatorname{Pr}=0.71, G r=2$ and $G m=2$.

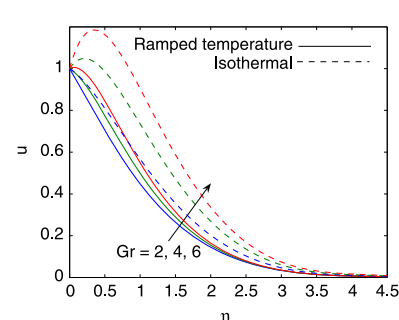

(a)

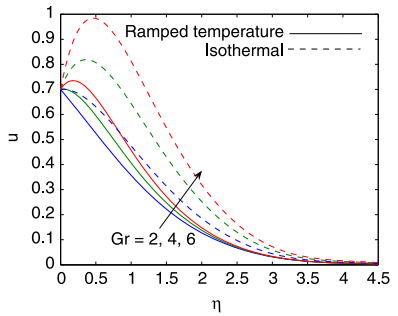

(b)

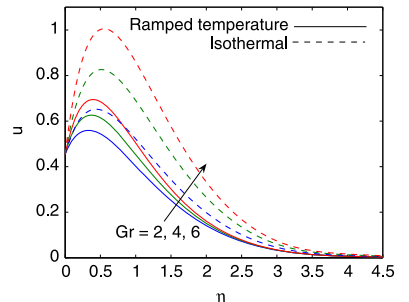

(c)

Figure 9 Effect of variation in the thermal Grashof number $G r$ on the fluid velocity $u(\eta, t)$ when (a) $f(t)=H(t)$, (b) $f(t)=t H(t)$, and (c) $f(t)=\cos \omega t H(t)$ for $t=0.7, S c=0.6, \phi=1, \operatorname{Pr}=0.71, M=3$ and $G m=2$.

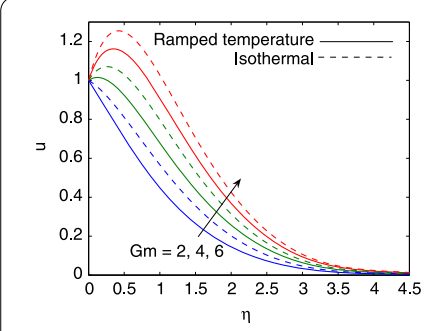

(a)

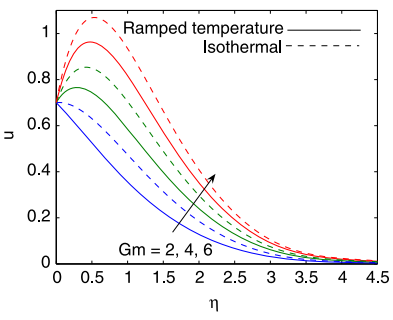

(b)

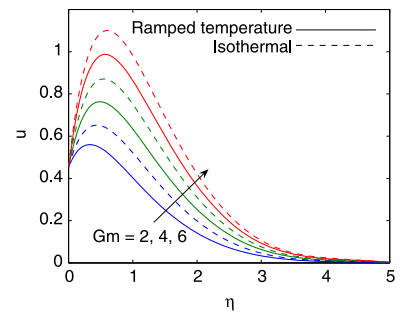

(c)

Figure 10 Effect of variation in the mass Grashof number $G m$ on the fluid velocity $u(\eta, t)$ when (a) $f(t)=H(t)$, (b) $f(t)=t H(t)$, and (c) $f(t)=\cos \omega t H(t)$ for $t=0.7, S c=0.6, \phi=1, \operatorname{Pr}=0.71, M=3$ and $G r=2$.

Figure 3 shows that for both ramped temperature and isothermal plates, the fluid temperature $T(\eta, t)$ increases with $t$ and decreases with an increase in either $\phi$ or $\operatorname{Pr}$. Since the Prandtl number $P r$ is the ratio of viscosity to the thermal diffusivity, an increase in $P r$ implies a decrease in thermal diffusivity. This implies that heat absorption tends to reduce the fluid temperature, whereas thermal diffusion and time have the opposite effect. The finding that the fluid temperature increases with thermal diffusion and time is in agreement with earlier results of Seth and Ansari [23] and Seth et al. [24].

Figure 4 shows that for both ramped temperature and isothermal plates, the fluid velocity $u(\eta, t)$ increases with an increase in $t$ in all cases except in the case of periodic acceleration of the plate. In this case, the fluid velocity near the plate decreases with an increase 
Table 1 Skin friction when $f(t)=H(t)$ for $S c=0.6$

\begin{tabular}{llllllrr}
\hline $\boldsymbol{t}$ & $\boldsymbol{\phi}$ & $\boldsymbol{P r}$ & $\boldsymbol{M}$ & $\boldsymbol{G r}$ & $\mathbf{G m}$ & \multicolumn{1}{c}{$\boldsymbol{\tau}_{\mathbf{1}}$} & \multicolumn{1}{c}{$\boldsymbol{\tau}_{\mathbf{i}}$} \\
\hline $\mathbf{0 . 3}$ & 1 & 0.71 & 3 & 2 & 2 & -0.80740428 & -0.06468570 \\
$\mathbf{0 . 5}$ & 1 & 0.71 & 3 & 2 & 2 & -0.49752200 & 0.17704678 \\
$\mathbf{0 . 7}$ & 1 & 0.71 & 3 & 2 & 2 & -0.28731934 & 0.25326881 \\
0.7 & $\mathbf{1}$ & 0.71 & 3 & 2 & 2 & -0.28731934 & 0.25326881 \\
0.7 & $\mathbf{3}$ & 0.71 & 3 & 2 & 2 & -0.29496426 & -0.03525641 \\
0.7 & $\mathbf{5}$ & 0.71 & 3 & 2 & 2 & -0.31937448 & -0.09571177 \\
0.7 & 1 & $\mathbf{0 . 5 0}$ & 3 & 2 & 2 & -0.26830104 & 0.26126499 \\
0.7 & 1 & $\mathbf{0 . 7 1}$ & 3 & 2 & 2 & -0.28731934 & 0.25326881 \\
0.7 & 1 & $\mathbf{7 . 0 0}$ & 3 & 2 & 2 & -0.43648929 & -0.25489651 \\
0.7 & 1 & 0.71 & $\mathbf{3}$ & 2 & 2 & -0.28731934 & 0.25326881 \\
0.7 & 1 & 0.71 & $\mathbf{5}$ & 2 & 2 & -1.01876598 & -0.61694534 \\
0.7 & 1 & 0.71 & $\mathbf{7}$ & 2 & 2 & -1.58008231 & -1.25625623 \\
0.7 & 1 & 0.71 & 3 & $\mathbf{2}$ & 2 & -0.28731934 & 0.25326881 \\
0.7 & 1 & 0.71 & 3 & $\mathbf{4}$ & 2 & 0.06194951 & 1.14312581 \\
0.7 & 1 & 0.71 & 3 & $\mathbf{6}$ & 2 & 0.41121836 & 2.03298282 \\
0.7 & 1 & 0.71 & 3 & 2 & $\mathbf{2}$ & -0.28731934 & 0.25326881 \\
0.7 & 1 & 0.71 & 3 & 2 & $\mathbf{4}$ & 0.82070361 & 1.36129176 \\
0.7 & 1 & 0.71 & 3 & 2 & $\mathbf{6}$ & 1.92872655 & 2.46931471 \\
\hline
\end{tabular}

Table 2 Skin friction when $f(t)=t H(t)$ for $S c=0.6$

\begin{tabular}{llllllrr}
\hline $\boldsymbol{t}$ & $\boldsymbol{\phi}$ & $\boldsymbol{P r}$ & $\boldsymbol{M}$ & $\boldsymbol{G r}$ & $\boldsymbol{G m}$ & \multicolumn{1}{c}{$\boldsymbol{\tau}_{\mathbf{2}}$} & \multicolumn{1}{c}{$\boldsymbol{\tau}_{\mathbf{2 i}}$} \\
\hline $\mathbf{0 . 3}$ & 1 & 0.71 & 3 & 2 & 2 & 0.24349996 & 0.98621853 \\
$\mathbf{0 . 5}$ & 1 & 0.71 & 3 & 2 & 2 & 0.12077153 & 0.79534031 \\
$\mathbf{0 . 7}$ & 1 & 0.71 & 3 & 2 & 2 & -0.04094174 & 0.49964642 \\
0.7 & $\mathbf{1}$ & 0.71 & 3 & 2 & 2 & -0.04094174 & 0.49964642 \\
0.7 & $\mathbf{3}$ & 0.71 & 3 & 2 & 2 & -0.04858666 & 0.21112119 \\
0.7 & $\mathbf{5}$ & 0.71 & 3 & 2 & 2 & -0.07299688 & 0.15066583 \\
0.7 & 1 & $\mathbf{0 . 5 0}$ & 3 & 2 & 2 & -0.02192344 & 0.50764260 \\
0.7 & 1 & $\mathbf{0 . 7 1}$ & 3 & 2 & 2 & -0.04094174 & 0.49964642 \\
0.7 & 1 & $\mathbf{7 . 0 0}$ & 3 & 2 & 2 & -0.19011168 & -0.00851890 \\
0.7 & 1 & 0.71 & $\mathbf{3}$ & 2 & 2 & -0.04094174 & 0.49964642 \\
0.7 & 1 & 0.71 & $\mathbf{5}$ & 2 & 2 & -0.56908866 & -0.16726802 \\
0.7 & 1 & 0.71 & $\mathbf{7}$ & 2 & 2 & -0.97488806 & -0.65106197 \\
0.7 & 1 & 0.71 & 3 & $\mathbf{2}$ & 2 & -0.04094174 & 0.49964642 \\
0.7 & 1 & 0.71 & 3 & $\mathbf{4}$ & 2 & 0.30832712 & 1.38950342 \\
0.7 & 1 & 0.71 & 3 & $\mathbf{6}$ & 2 & 0.65759597 & 2.27936043 \\
0.7 & 1 & 0.71 & 3 & 2 & $\mathbf{2}$ & -0.04094174 & 0.49964642 \\
0.7 & 1 & 0.71 & 3 & 2 & $\mathbf{4}$ & 1.06708121 & 1.60766936 \\
0.7 & 1 & 0.71 & 3 & 2 & $\mathbf{6}$ & 2.17510416 & 2.71569231 \\
\hline
\end{tabular}

in $t$ and thereafter attains its usual nature, which is to increase with $t$. Thus, in general, it may be concluded that the fluid velocity in all three cases of interest increases with time. Figures 5-10 show that for both ramped temperature and isothermal plates, in all three cases, the fluid velocity decreases with an increase in $S c, \phi, P r$, or $M$, whereas it increases with an increase in Gr or Gm. Thus it follows that heat absorption and the magnetic field tend to retard the fluid flow, whereas thermal diffusion, mass diffusion, thermal buoyancy force and mass buoyancy force have the opposite effect. We further note from Figures 4-10 that the fluid velocity in the case of a flow past an isothermal plate is higher than that of a flow past a ramped temperature plate. The effect of the magnetic field, thermal buoyancy force and time on the fluid velocity in the case of a flow past an impulsively started plate are in agreement with that of Seth et al. [24].

Tables 1-3 show that the skin friction decreases with an increase in time $t$ except for the case where the plate moves with periodic acceleration. In this case, the skin friction 
Table 3 Skin friction when $f(t)=\cos \omega t H(t)$ for $S c=0.6$

\begin{tabular}{llllllll}
\hline $\boldsymbol{t}$ & $\boldsymbol{\phi}$ & $\boldsymbol{P r}$ & $\boldsymbol{M}$ & $\mathbf{G r}$ & $\mathbf{G m}$ & $\boldsymbol{\tau}_{\mathbf{3}}$ & $\boldsymbol{\tau}_{\mathbf{3 i}}$ \\
\hline $\mathbf{0 . 3}$ & 1 & 0.71 & 3 & 2 & 2 & -0.51381907 & 0.22889951 \\
$\mathbf{0 . 5}$ & 1 & 0.71 & 3 & 2 & 2 & 0.23463588 & 0.90920466 \\
$\mathbf{0 . 7}$ & 1 & 0.71 & 3 & 2 & 2 & 0.98010460 & 1.52069275 \\
0.7 & $\mathbf{1}$ & 0.71 & 3 & 2 & 2 & 0.98010460 & 1.52069275 \\
0.7 & $\mathbf{3}$ & 0.71 & 3 & 2 & 2 & 0.97245968 & 1.23216753 \\
0.7 & $\mathbf{5}$ & 0.71 & 3 & 2 & 2 & 0.94804946 & 1.17171216 \\
0.7 & 1 & $\mathbf{0 . 5 0}$ & 3 & 2 & 2 & 0.99912289 & 1.52868893 \\
0.7 & 1 & $\mathbf{0 . 7 1}$ & 3 & 2 & 2 & 0.98010460 & 1.52069275 \\
0.7 & 1 & $\mathbf{7 . 0 0}$ & 3 & 2 & 2 & 0.83093465 & 1.01252743 \\
0.7 & 1 & 0.71 & $\mathbf{3}$ & 2 & 2 & 0.98010460 & 1.52069275 \\
0.7 & 1 & 0.71 & $\mathbf{5}$ & 2 & 2 & 0.49014643 & 0.89196706 \\
0.7 & 1 & 0.71 & $\mathbf{7}$ & 2 & 2 & 0.11796087 & 0.44178695 \\
0.7 & 1 & 0.71 & 3 & $\mathbf{2}$ & 2 & 0.98010460 & 1.52069275 \\
0.7 & 1 & 0.71 & 3 & $\mathbf{4}$ & 2 & 1.32937345 & 2.41054975 \\
0.7 & 1 & 0.71 & 3 & $\mathbf{6}$ & 2 & 1.67864230 & 3.30040676 \\
0.7 & 1 & 0.71 & 3 & 2 & $\mathbf{2}$ & 0.98010460 & 1.52069275 \\
0.7 & 1 & 0.71 & 3 & 2 & $\mathbf{4}$ & 2.08812754 & 2.62871570 \\
0.7 & 1 & 0.71 & 3 & 2 & $\mathbf{6}$ & 3.19615049 & 3.73673864 \\
\hline
\end{tabular}

Table 4 The Nusselt number and Sherwood number

\begin{tabular}{lllllll}
\hline $\boldsymbol{t}$ & $\boldsymbol{\phi}$ & $\boldsymbol{P r}$ & $\boldsymbol{S c}$ & $\mathbf{N u}$ & $\mathbf{N} \boldsymbol{u}_{\boldsymbol{i}}$ & $\boldsymbol{S h}$ \\
\hline $\mathbf{0 . 3}$ & 1 & 0.71 & 0.6 & 0.57134752 & 1.11605411 & 0.79788456 \\
$\mathbf{0 . 5}$ & 1 & 0.71 & 0.6 & 0.77913255 & 0.98302070 & 0.61803872 \\
$\mathbf{0 . 7}$ & 1 & 0.71 & 0.6 & 0.96929143 & 0.92531051 & 0.52233806 \\
0.7 & $\mathbf{1}$ & 0.71 & 0.6 & 0.96929143 & 0.92531051 & - \\
0.7 & $\mathbf{3}$ & 0.71 & 0.6 & 1.26243402 & 1.47003548 & - \\
0.7 & $\mathbf{5}$ & 0.71 & 0.6 & 1.50704023 & 1.88594507 & - \\
0.7 & 1 & $\mathbf{0 . 5 0}$ & 0.6 & 0.81341130 & 0.77650333 & - \\
0.7 & 1 & $\mathbf{0 . 7 1}$ & 0.6 & 0.96929143 & 0.92531051 & - \\
0.7 & 1 & $\mathbf{7 . 0 0}$ & 0.6 & 3.04350641 & 2.90540943 & - \\
0.7 & 1 & 0.71 & $\mathbf{0 . 4 0}$ & - & - & 0.42648724 \\
0.7 & 1 & 0.71 & $\mathbf{0 . 6 0}$ & - & - & 0.52233806 \\
0.7 & 1 & 0.71 & $\mathbf{0 . 7 8}$ & - & - & 0.59555702 \\
\hline
\end{tabular}

for both ramped temperature and isothermal plates increases with an increase in time. It is evident that the skin friction, for both ramped temperature and isothermal plates, in all cases, increases with $\phi, P r$, or $M$, whereas it decreases with an increase in $G r$ or $G m$, which implies that heat absorption and the magnetic field tend to increase the shear stress at the plate, while the thermal diffusion, thermal buoyancy force and mass buoyancy force have the reverse effect on it. The effects of the magnetic field, thermal buoyancy force and time on the skin friction in the case of a flow past an impulsively moving plate are similar to those obtained by Seth et al. [24].

In Table 4 we note that the Nusselt number for both ramped temperature and isothermal plates increases with $\operatorname{Pr}, \phi$ or $t$, which implies that heat absorption and time have a tendency to enhance the rate of heat transfer at the plate, whereas thermal diffusion has the reverse effect on it. The tendency of the Nusselt number to increase with time is in agreement with the results of Seth et al. [24]. It is also observed from Table 4 that the Sherwood number decreases with an increase in $t$, whereas it increases with an increase in $S c$. Thus both mass diffusivity and time tend to reduce the rate of mass transfer at the plate.

To analyze the behavior of species concentration, fluid temperature and fluid velocity for large time $t$, the profiles of species concentration and temperature are presented in Fig- 


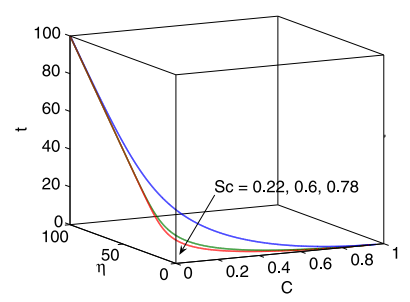

(a)

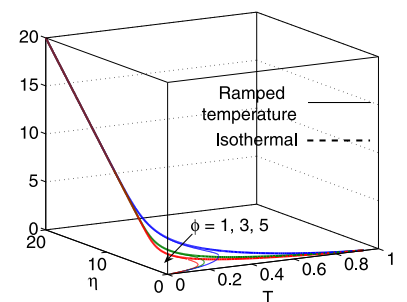

(b)

Figure 11 Behavior of (a) $C(\eta, t)$ and (b) $T(\eta, t)$ for large time $t$.

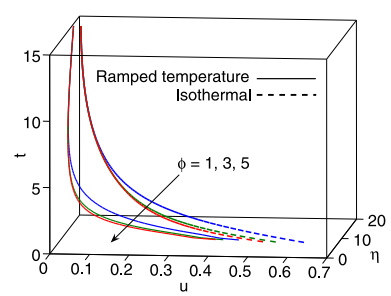

(a)

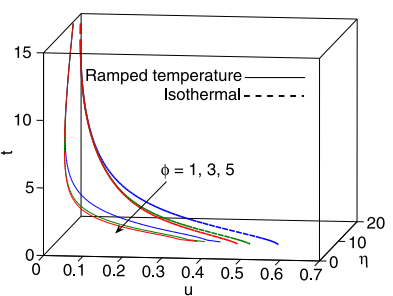

(b)

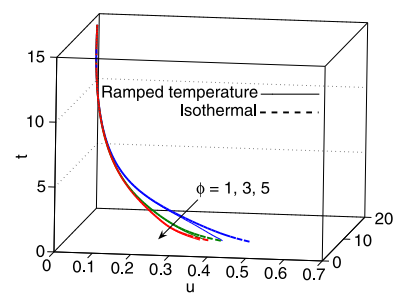

(c)

Figure 12 Behavior of $u(\eta, t)$ for large time $t$ when (a) $f(t)=H(t),(b) f(t)=t H(t)$, and (c) $f(t)=\cos \omega t H(t)$.

ure 11 and the velocity profiles are depicted in Figure 12. Figure 11 shows that the unsteady nature of species concentration and fluid temperature last even for large values of time $t$, which implies that even for large values of time, the species concentration and temperature field does not approach the steady state. However, in Figure 12, it may be seen that the velocity profiles for all the cases of plate movement become parallel to the $t$-axis after a certain time. This shows that the velocity field approaches steady state as $t \rightarrow \infty$. It is also observed that the velocity field approaches the steady state much faster in the case of impulsive and accelerated movements of the plate as compared to periodic acceleration of the plate.

\section{Conclusions}

We have investigated the unsteady MHD free convection heat and mass transfer flow of a viscous, incompressible, electrically conducting and heat absorbing fluid flow past an infinite vertical flat plate. The flow was induced by a time-dependent movement of the flat plate. Three cases of particular interest, namely (1) movement of the plate with uniform velocity, (2) movement of the plate with single acceleration and (3) movement of the plate with periodic acceleration, have been discussed. Exact solutions of the governing equations were found using Laplace transforms. The important findings may be summarized as follows:

- the mass diffusion rate and time tend to increase species concentration,

- heat absorption reduces the fluid temperature, whereas thermal diffusion and time have the opposite effect,

- heat absorption and the magnetic field tend to retard the fluid flow, whereas thermal diffusion, mass diffusion, thermal buoyancy force and mass buoyancy force have the opposite effect, 
- heat absorption and the magnetic field tend to increase the shear stress at the plate, whereas thermal diffusion, thermal buoyancy force and mass buoyancy force have the reverse effect,

- heat absorption and time tend to increase the rate of heat transfer at the plate, whereas thermal diffusion has the reverse effect, and

- mass diffusivity and time tend to reduce the rate of mass transfer at the plate.

\section{Competing interests}

The authors declare that they have no competing interests.

\section{Authors' contributions}

All the authors contributed equally in the preparation of the manuscript. All the authors read and approved the final version of the manuscript.

\section{Author details}

'School of Mathematics, Statistics and Computer Science, University of KwaZulu-Natal, Scottsville, Pietermaritzburg, 3209, South Africa. ${ }^{2}$ Department of Mathematics, School of Applied Sciences, KIIT University, Bhubaneswar, 751024, India.

\section{Acknowledgements}

The authors sincerely thank the reviewers for their valuable comments which helped to improve the quality of the paper. $\mathrm{RN}$ is grateful to the University of KwaZulu-Natal for financial support.

Received: 5 March 2013 Accepted: 16 October 2013 Published: 20 Nov 2013

\section{References}

1. Hayat, T, Nadeem, S, Asghar, S: MHD rotating flow of a third-grade fluid on an oscillating porous plate. Acta Mech. $152,177-190(2001)$

2. Hayat, T, Nadeem, S, Siddiqui, AM, Asghar, S: An oscillating hydromagnetic non-Newtonian flow in a rotating system. Appl. Math. Lett. 17, 609-614 (2004)

3. Seth, GS, Nandkeolyar, R, Ansari, MS: Hartmann flow in a rotating system in the presence of inclined magnetic field with Hall effects. Tamkang J. Sci. Eng. 13(3), 243-252 (2010)

4. Kim, YJ: Unsteady MHD convective heat transfer past a semi-infinite vertical porous moving plate with variable suction. Int. J. Eng. Sci. 38, 833-845 (2000)

5. Takhar, HS, Roy, S, Nath, G: Unsteady free convection flow of an infinite vertical porous plate due to the combined effects of thermal and mass diffusion, magnetic field and Hall currents. Heat Mass Transf. 39, 825-834 (2003)

6. Ahmed, N, Sarmah, HK, Kalita, D: Thermal diffusion effect on a three-dimensional MHD free convection with mass transfer flow from a porous vertical plate. Lat. Am. Appl. Res. 41, 165-176 (2011)

7. Hossain, MA, Mandal, AC: Mass transfer effects on the unsteady hydromagnetic free convection flow past an accelerated vertical porous plate. J. Phys. D, Appl. Phys. 18, 163-169 (1985)

8. Jha, BK: MHD free convection and mass transfer flow through a porous medium. Astrophys. Space Sci. 175, 283-289 (1991)

9. Elbashbeshy, EMA: Heat and mass transfer along a vertical plate with variable surface tension and concentration in the presence of magnetic field. Int. J. Eng. Sci. 34, 515-522 (1997)

10. Chamkha, AJ, Khaled, ARA: Hydromagnetic combined heat and mass transfer by natural convection from a permeable surface embedded in a fluid satural porous medium. Int. J. Numer. Methods Heat Fluid Flow 10(5), 455-476 (2000)

11. Chen, $\mathrm{CH}$ : Combined heat and mass transfer in MHD free convection from a vertical surface with Ohmic heating and viscous dissipation. Int. J. Eng. Sci. 42,699-713 (2004)

12. Afify, AA: Similarity solution in MHD: effects of thermal diffusion and diffusion thermo on free convective heat and mass transfer over a stretching surface considering suction or injection. Commun. Nonlinear Sci. Numer. Simul. 14, 2202-2214 (2009)

13. Eldabe, NTM, Elbashbeshy, EA, Elsayed, MA, Hasanin, WSA, Elsaid, EM: Unsteady motion of MHD viscous incompressible fluid with heat and mass transfer through porous medium near a moving vertical plate. Int. J. Energy Technol. 3, 1-11 (2011)

14. Chamkha, AJ, Khaled, ARA: Similarity solutions for hydromagnetic simultaneous heat and mass transfer by natural convection from an inclined plate with heat generation or absorption. Heat Mass Transf. 37, 117-123 (2001)

15. Kamel, MH: Unsteady MHD convection through porous medium with combined heat and mass transfer with heat source/sink. Energy Convers. Manag. 42, 393-405 (2001)

16. Chamkha, AJ: Unsteady MHD convective heat and mass transfer past a semi-infinite vertical permeable moving plate with heat absorption. Int. J. Eng. Sci. 42, 217-230 (2004)

17. Makinde, OD: On MHD boundary-layer flow and mass transfer past a vertical plate in a porous medium with constant heat flux. Int. J. Numer. Methods Heat Fluid Flow 19(3/4), 546-554 (2009)

18. Hayday, AA, Bowlus, DA, McGraw, RA: Free convection from a vertical plate with step discontinuities in surface temperature. J. Heat Transf. 89, 244-250 (1967)

19. Kelleher, M: Free convection from a vertical plate with discontinuous wall temperature. J. Heat Transf. 93, 349-356 (1971)

20. Kao, TT: Laminar free convective heat transfer response along a vertical flat plate with step jump in surface temperature. Lett. Heat Mass Transf. 2, 419-428 (1975) 
21. Lee, S, Yovanovich, MM: Laminar natural convection from a vertical plate with a step change in wall temperature. J. Heat Transf. 113, 501-504 (1991)

22. Chandran, P, Sacheti, NC, Singh, AK: Natural convection near a vertical plate with ramped wall temperature. Heat Mass Transf. 41, 459-464 (2005)

23. Seth, GS, Ansari, MS: MHD natural convection flow past an impulsively moving vertical plate with ramped wall temperature in the presence of thermal diffusion with heat absorption. Int. J. Appl. Mech. Eng. 15, 199-215 (2010)

24. Seth, GS, Ansari, MS, Nandkeolyar, R: MHD natural convection flow with radiative heat transfer past an impulsively moving plate with ramped wall temperature. Heat Mass Transf. 47, 551-561 (2011)

25. Cramer, K, Pai, S: Magnetofluiddynamics for Engineers and Applied Physicists. McGraw-Hill, New York (1973)

26. Meyer, RC: On reducing aerodynamic heat-transfer rates by magnetohydrodynamic techniques. J. Aeronaut. Sci. 25, 561-572(1958)

10.1186/1687-2770-2013-247

Cite this article as: Nandkeolyar et al.: Exact solutions of unsteady MHD free convection in a heat absorbing fluid

flow past a flat plate with ramped wall temperature. Boundary Value Problems 2013, 2013:247

\section{Submit your manuscript to a SpringerOpen ${ }^{\circ}$ journal and benefit from:}

- Convenient online submission

- Rigorous peer review

Immediate publication on acceptance

Open access: articles freely available online

High visibility within the field

- Retaining the copyright to your article 